\title{
ADDENDUM
}

\section{INTEGRABILITY OF OPEN SPIN CHAINS WITH QUANTUM ALGEBRA SYMMETRY}

[INT. J. MOD. PHYS. A, Vol. 6, 5231 (1991)]

Luca Mezincescu and Rafael I. Nepomechie

Department of Physics

University of Miami, Coral Gables, FL 33124

\begin{abstract}
In the above reference, we prove that certain quantum spin chains with quantumalgebra symmetry are integrable. Specifically, these are the quantum-algebra-invariant open chains associated with the affine Lie algebras $A_{1}^{(1)}, A_{2 n}^{(2)}, A_{2 n-1}^{(2)}, B_{n}^{(1)}, C_{n}^{(1)}$, and $D_{n}^{(1)}$ in the fundamental representation. Conspicuously absent from this list is $A_{n}^{(1)}$ for $n>1$. This is because in order to demonstrate integrability, we assume that the corresponding $R$ matrix has crossing symmetry, which is not true in the case $A_{n}^{(1)}$ for $n>1$.

Sklyanin has demonstrated ${ }^{1}$ that an integrable open spin chain can be constructed with an $R$ matrix that satisfies nothing more than the Yang-Baxter equation. In particular, no assumption of crossing symmetry is necessary. This suggests that the quantum-algebrainvariant open chain associated with $A_{n}^{(1)}$ for $n>1$ may be integrable. In this Addendum, we show that this is in fact the case.
\end{abstract}

Let $R(u)$ be a solution of the Yang-Baxter equation corresponding to a non-exceptional affine Lie algebra $g^{(k)}$ in the fundamental representation. According to Bazhanov ${ }^{2}$ and Jimbo $^{3}$, such an $R$ matrix has $P T$ symmetry

$$
\mathcal{P}_{12} R_{12}(u) \mathcal{P}_{12} \equiv R_{21}(u)=R_{12}(u)^{t_{1} t_{2}}
$$


and is unitary

$$
R_{12}(u) R_{21}(-u)=\zeta(u)
$$

where $\zeta(u)$ is some even scalar function of $u$. Moreover, according to Reshetikhin and Semenov-Tian-Shansky ${ }^{4}$, there exists a matrix $M$ such that

$$
\left\{\left\{\left\{R_{12}(u)^{t_{2}}\right\}^{-1}\right\}^{t_{2}}\right\}^{-1}=\frac{\zeta(u+\rho)}{\zeta(u+2 \rho)} M_{2} R_{12}(u+2 \rho) M_{2}^{-1} .
$$

Also, $M^{t}=M$ and

$$
[R(u), M \otimes M]=0
$$

We do not assume that $R$ has crossing symmetry.

Let us introduce matrices $K^{-}(u)$ and $K^{+}(u)$ as before ${ }^{5}$ :

$$
R_{12}(u-v) K_{1}^{-}(u) R_{21}(u+v) K_{2}^{-}(v)=K_{2}^{-}(v) R_{12}(u+v) K_{1}^{-}(u) R_{21}(u-v)
$$

and

$$
\begin{aligned}
& R_{12}(-u+v) K_{1}^{+}(u)^{t_{1}} M_{1}^{-1} R_{21}(-u-v-2 \rho) M_{1} K_{2}^{+}(v)^{t_{2}} \\
& \quad=K_{2}^{+}(v)^{t_{2}} M_{1} R_{12}(-u-v-2 \rho) M_{1}^{-1} K_{1}^{+}(u)^{t_{1}} R_{21}(-u+v) .
\end{aligned}
$$

Sklyanin $^{1}$ has found a more general set of relations, which reduces to this set when $R$ satisfies Eqs. (1) and (2).

With the help of Eqs. (3) and (4), one can verify that

$$
K^{+}(u)=K^{-}(-u-\rho)^{t} M
$$

is an automorphism. For all cases except $D_{n}^{(2)}$ (so, in particular, for the case $A_{n}^{(1)}$ ),

$$
K^{-}(u)=1
$$

is a solution of Eq. (5). Hence,

$$
K^{+}(u)=M
$$


is a solution of Eq. (6). And hence, the corresponding commuting transfer matrix is ${ }^{6,5}$

$$
t(u)=\operatorname{tr} K^{+}(u) T(u) K^{-}(u) T(-u)^{-1}=\operatorname{tr} M T(u) T(-u)^{-1}
$$

This transfer matrix commutes also with the generators of the quantum algebra $U_{q}\left[g_{0}\right]$, where $g_{0}$ is the maximal finite-dimensional subalgebra of $g^{(k)}$. Indeed, let us recall that the monodromy matrix $T(u)$, upon suitably taking the limit $u \rightarrow \pm \infty$, becomes an upper or lower triangular matrix $T_{ \pm}$whose entries are elements of $U_{q}\left[g_{0}\right]$. The proof of the formula

$$
\left[T_{ \pm}, t(u)\right]=0
$$

is essentially the one given (following Kulish and Sklyanin ${ }^{7}$ ) in Ref. 8.

In conclusion, the case $A_{n}^{(1)}$ can be treated in almost the same way as the other cases which we have previously studied. The only difference is that the crossing relation for the $R$ matrix is replaced by the weaker relation (3).

A number of interesting questions remain:

1. One may try to extend this analysis to the case $D_{n}^{(2)}$, for which Eq. (8) does not hold. Moreover, the exceptional cases have not yet been investigated.

2. One may try to solve the $A_{n}^{(1)}$ chains by the analytical Bethe Ansatz. This would require an extension of the known ${ }^{9,10}$ formalism, which assumes crossing symmetry.

3. One may further explore ${ }^{1}$ open chains associated with a matrix $R(u)$ which does not have PT symmetry, or with a matrix $R(u, v)$ which does not depend on the difference $u-v$. The chiral Potts model is presumably an example of the second type.

We are indebted to E.K. Sklyanin for sharing with us his unpublished work ${ }^{1}$. We also thank N. Yu. Reshetikhin for a discussion on the scalar factor in Eq. (3). This work was supported in part by the National Science Foundation under Grant No. PHY-90 07517. 


\section{References}

1. E.K. Sklyanin, unpublished.

2. V.V. Bazhanov, Phys. Lett. $159 B$ (1985) 321; Commun. Math. Phys. 113 (1987) 471.

3. M. Jimbo, Commun. Math. Phys. 102 (1986) 537; Lecture Notes in Physics, Vol. 246 (Springer, 1986) 335 .

4. N. Yu. Reshetikhin and M.A. Semenov-Tian-Shansky, Lett. Math. Phys. 19 (1990) 133. See also I. Frenkel and N. Yu. Reshetikhin, preprint.

5. L. Mezincescu and R.I. Nepomechie, J. Phys. A24 (1991) L17.

6. E.K. Sklyanin, J. Phys. A21 (1988) 2375.

7. P.P. Kulish and E.K. Sklyanin, J. Phys. A24 (1991) L435.

8. L. Mezincescu and R.I. Nepomechie, Mod. Phys. Lett. A6 (1991) 2497.

9. N. Yu. Reshetikhin, Lett. Math. Phys. 14 (1987) 235.

10. L. Mezincescu and R.I. Nepomechie, Nucl. Phys. B372 (1992) 597. 